\title{
Nanoemulsion-based gel formulations of COX-2 inhibitors for enhanced efficacy in inflammatory conditions
}

\author{
R. R. Lala $\cdot$ N. G. Awari
}

Received: 1 September 2012/ Accepted: 8 November 2012/Published online: 19 January 2013

(C) The Author(s) 2013. This article is published with open access at Springerlink.com

\begin{abstract}
In the present study, we have investigated the potential of a nanoemulsion (thermodynamically stable transparent dispersions of oil and water having a droplet size $<200 \mathrm{~nm}$ ) formulation for the topical delivery of COX-2 inhibitors using etoricoxib as a model drug. Various oil-in-water nanoemulsions were prepared by the spontaneous emulsification method. The nanoemulsion area was identified by constructing pseudo-ternary phase diagrams. The prepared nanoemulsions were subjected to thermodynamic stability testing. Those that passed these tests were characterized for viscosity, droplet size and differential scanning calorimetry. Topical permeation of etoricoxib through porcine abdominal skin was estimated using the Franz diffusion cell. The ex vivo skin permeation profile of optimized formulations was compared with that of etoricoxib conventional gel. A significant increase in permeability was observed in optimized nanoemulsion formulations consisting of $2 \% \mathrm{w} / \mathrm{w}$ of etoricoxib, $20 \%$ $\mathrm{w} / \mathrm{w}$ of Triacetin, $38 \% \mathrm{w} / \mathrm{w}$ of a surfactant mixture (Cremophor RH 40:Transcutol P), and $42 \% \mathrm{w} / \mathrm{w}$ of water. The anti-inflammatory effects of this formulation on carrageenan-induced paw edema in rats showed a significant increase in the percent inhibition value $(84.61 \%$ with the nanoemulsion gel and $92.30 \%$ with the nanoemulsion) as compared with the conventional gel $(69.23 \%)$ after $6 \mathrm{~h}$ when compared with etoricoxib conventional gel. These results suggest that nanoemulsions can serve as potential
\end{abstract}

R. R. Lala $(\bowtie) \cdot$ N. G. Awari

Department of Pharmaceutics, Prin. K. M. Kundnani College

of Pharmacy, 23, Jote Joy Building, Rambhau Salgaonkar Marg,

Cuffe Parade, Mumbai, MH 400005, India

e-mail: r_r_lala@yahoo.co.in

N. G. Awari

e-mail: nileshgaawari@yahoo.in vehicles for improved transdermal delivery of antiinflammatory agents such as etoricoxib.

Keywords Etoricoxib - Nanoemulsion-based gels · Topical delivery · Anti-inflammatory effects .

Pseudo-ternary phase diagram

\section{Introduction}

Nonsteroidal anti-inflammatory drugs (NSAIDs) are the most commonly used drugs to reduce pain and inflammation (Escribano et al. 2003; Lee et al. 2003). COX-2 inhibitors that have been introduced in medical practice are recommended orally for patients with chronic inflammatory degenerative diseases such as rheumatoid arthritis and osteoarthritis ( $\mathrm{Li}$ et al. 2005), and also as anti-inflammatory, antipyretic, and analgesic agents (Yang et al. 2002). The chronic oral administration of these causes gastrointestinal ulcers, bleeding and adverse cardiovascular events (Lehmann et al. 2001; Yamazaki et al. 1997). Use of the transdermal route eliminates these side effects, increases patient compliance, avoids first-pass metabolism, and maintains the plasma drug levels (Gaurel et al. 1997). Therefore, an improved nanoemulsion formulation of COX-2 inhibitors such as etoricoxib with a high degree of permeation could be useful in the treatment of locally inflamed skin and inflammatory and painful states of supporting structures of the body, such as bones, ligaments, joints, tendons, and muscles (Alvarez-Figueroa and Blanco-Mendez 2001). There has been an increased interest during recent years in the use of topical vehicle systems that could modify drug permeation through the skin. Many of the dermal vehicles contain chemical enhancers and solvents to achieve these goals (Peltola et al. 2003; Walters 
et al. 1989). But use of these chemical enhancers may be harmful, especially on chronic application, as many of them are irritants. Therefore, it is desirable to develop a topical vehicle system that does not require the use of chemical enhancers to facilitate drug permeation through the skin. One of the most promising techniques for enhancement of transdermal permeation of drugs are nanoemulsions and nanoemulsion gels. These are thermodynamically stable transparent (translucent) dispersions of oil and water stabilized by an interfacial film of surfactant and cosurfactant molecules having a droplet size of less than $200 \mathrm{~nm}$ (Shafiq et al. 2000; Shafiq et al. 2007; Rhee et al. 2001). Due to their characteristic size and properties, which include kinetic stability, they are very effective in enveloping and/or solubilizing drugs and successfully transferring them toward suitable targets. Many studies have shown that nanoemulsion formulations possess improved transdermal and dermal delivery properties for drugs in vitro, as well as in vivo over conventional topical formulations such as emulsions and gels (Gonzalez et al. 1994; Kemken et al. 1992; Osborne et al. 1991). This article describes the potential use of nanoemulsion-based gel systems for the transdermal delivery of etoricoxib using nonirritating, pharmaceutically acceptable ingredients without using additional permeation enhancers, since the excipients themselves act as permeation enhancers (Kreilgaard et al. 2000; Kriwet and MullerGoymann 1995; Trotta 1999).

\section{Experiment}

Materials and methods

Etoricoxib (EXB) was received as a gift sample from Genovo Development Services, Bangalore. Polyoxyethylene 80 sorbitan monooleate, sorbitan oleates, soyabean lecithin, polyoxyl 40 hydrogenated castor oil, diethylene glycol monoethyl ether, sodium lauryl sulfate, propylene glycol, polyethylene glycol (400, 600), Carbopol-934, Poloxamer 407 were obtained from Sigma Laboratories, Mumbai (India). Isopropyl myristate (IPM), oleic acid, glycerol triacetate (Triacetin), olive oil, were obtained from E-Merck (Mumbai, India). Polyoxy-35-castor oil (Cremophor RH 40) and diethylene glycol monoethyl ether (Transcutol P) were gift samples from Gattefosse, Cedex, France. All other chemicals used in the study were of analytical reagent grade.

Screening of excipients

Compatibility studies were carried out to investigate interactions between the drug and the polymers. Interaction studies were carried out by infrared spectroscopy and DSC.
The solubility of EXB in various oils (Triacetin, castor oil, oleic acid, IPM, and olive oil), surfactants (Polyoxyethylene 80 sorbitan monooleate, sorbitan oleates, polyoxyl 40 hydrogenated castor oil, diethylene glycol monoethyl ether, propylene glycol) was determined by dissolving an excess amount of EXB in $5 \mathrm{ml}$ of each of the selected oils, surfactants and cosurfactants. The vials were then kept at $37 \pm 1.0{ }^{\circ} \mathrm{C}$ for $72 \mathrm{~h}$ to equilibrate and centrifuged at 3,000 rpm for $15 \mathrm{~min}$. The supernatant was filtered through a $0.45-\mu \mathrm{m}$ membrane filter. The concentration of EXB was determined by a validated HPLC method using a UV detector at $284 \mathrm{~nm}$.

Preparation of nanoemulsions

Various formulations of nanoemulsions (using different concentrations of excipients) of EXB were prepared by the low energy emulsification method (aqueous titration method). Optimized formulations of nanoemulsions were prepared by dissolving $2 \% \mathrm{w} / \mathrm{w}$ of EXB in a $10-20 \% \mathrm{w} / \mathrm{w}$ mixture of the oily phase. Then, 30-45\% w/w mixture of Smix (surfactant:cosurfactant) was added slowly to the oil phase, followed by the slow addition of distilled water to adjust the final preparation to $100 \% \mathrm{w} / \mathrm{w}$. The composition of the various batches prepared is given in Table 1.

Construction of pseudo-ternary phase diagrams

On the basis of solubility studies, Triacetin was selected as the oil phase with high solubility of the drug $(96.84 \pm 1.89 \mathrm{mg} / \mathrm{ml})$. Cremophor RH 40 (HLB-14) and Transcutol P (HLB-4.2) were selected as surfactant and cosurfactant, respectively, since a stable, transparent nanoemulsion was obtained with them. Surfactant and cosurfactant (Smix) were mixed in various weight ratios

Table 1 Composition of various batches of nanoemulsion formulations

\begin{tabular}{lllll}
\hline Batch no. & Smix ratio & Oil phase $(\%)$ & Smix $(\%)$ & $\begin{array}{l}\text { Aqueous } \\
\text { phase }(\%)\end{array}$ \\
\hline F1 & $1: 0$ & 10 & 67 & 23 \\
F2 & $1: 0$ & 15 & 60 & 25 \\
F4 & $3: 1$ & 15 & 50 & 35 \\
F5 & $3: 1$ & 10 & 36 & 54 \\
F6 & $3: 1$ & 20 & 50 & 30 \\
F7 & $2: 1$ & 10 & 35 & 55 \\
F8 & $2: 1$ & 15 & 39 & 46 \\
F9 & $2: 1$ & 20 & 38 & 42 \\
F10 & $1: 1$ & 10 & 50 & 40 \\
F11 & $1: 1$ & 15 & 35 & 50 \\
F13 & $4: 1$ & 20 & 22 & 58 \\
F14 & $4: 1$ & 15 & 26 & 59 \\
\hline
\end{tabular}


(1:0, 1:2, 1:3, 1:1, 2:1, 3:1 and 4:1). These Smix ratios were selected in increasing concentrations of surfactant with respect to the cosurfactant and increasing concentration of cosurfactant with respect to surfactant for a detailed study of the phase diagrams as required for nanoemulsion formation. The physical state of the nanoemulsion was marked on a pseudo-3-component phase diagram with one axis representing the aqueous phase, second representing oil, and the third representing a mixture of surfactant and cosurfactant in fixed weight ratios (Fig. 1). Various combinations of oil and Smix (from 1:9 to 9:1) (Table 2) were prepared so that maximum ratios were covered for the study to delineate the boundaries of phases precisely formed in the phase diagrams. Pseudo-ternary phase diagrams of oil, Smix, and aqueous phase were developed using the aqueous titration method (Barzin and Sadatnia 2007). The aqueous phase was slowly titrated with the weight ratio of oil and Smix, and visual observations were made for transparent and easily flowable oil-in-water $(\mathrm{o} / \mathrm{w})$ nanoemulsions (Craig et al. 1995; Lawrence and Rees 2000).

Nanoemulsion Formulation

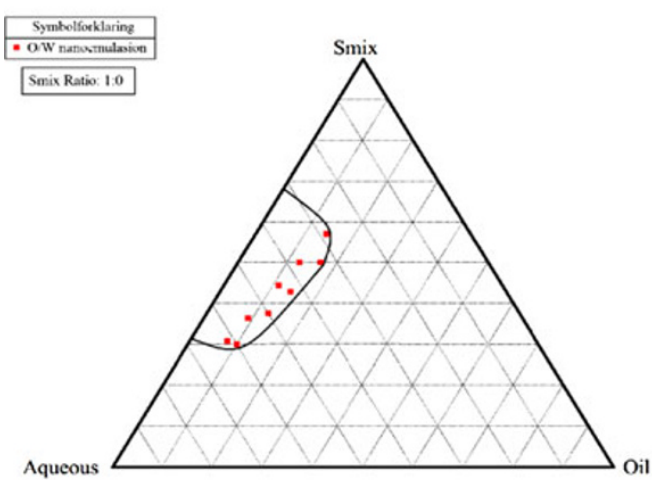

Nanoemulsion Formulation

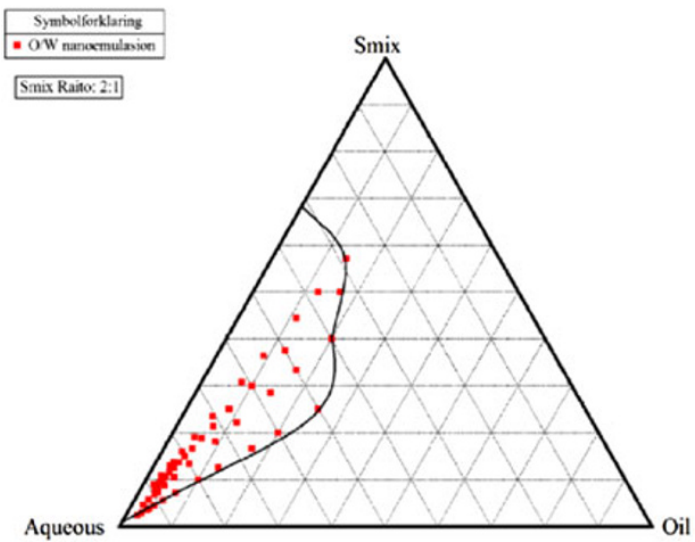

A

C
Table 2 Construction of pseudo-ternary diagram

\begin{tabular}{lll}
\hline Smix(1:2):oil & $\operatorname{Smix}(1: 1):$ oil & $\operatorname{Smix}(2: 1):$ oil \\
\hline $1: 9$ & $1: 9$ & $1: 9$ \\
$2: 8$ & $2: 8$ & $2: 8$ \\
$3: 7$ & $3: 7$ & $3: 7$ \\
$4: 6$ & $4: 6$ & $4: 6$ \\
$5: 5$ & $5: 5$ & $5: 5$ \\
$6: 4$ & $6: 4$ & $6: 4$ \\
$7: 3$ & $7: 3$ & $7: 3$ \\
$8: 2$ & $8: 2$ & $8: 2$ \\
$9: 1$ & $9: 1$ & $9: 1$ \\
\hline
\end{tabular}

Selection of nanoemulsion formulations

From each phase diagram constructed, different combinations were selected from the nanoemulsion region so that the drug etoricoxib $(2 \% \mathrm{w} / \mathrm{w})$ could be incorporated into the oil phase. Selected formulations were subjected to thermodynamic stability testing ( $\mathrm{Li}$ et al. 2005).

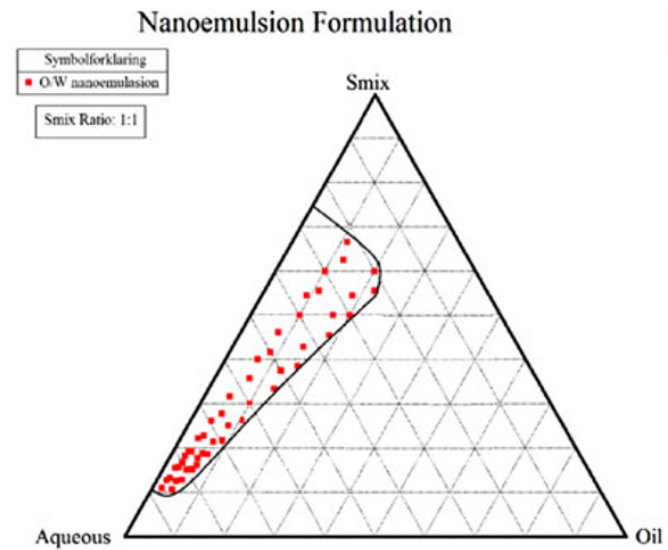

B

Nanoemulsion Formulation

D

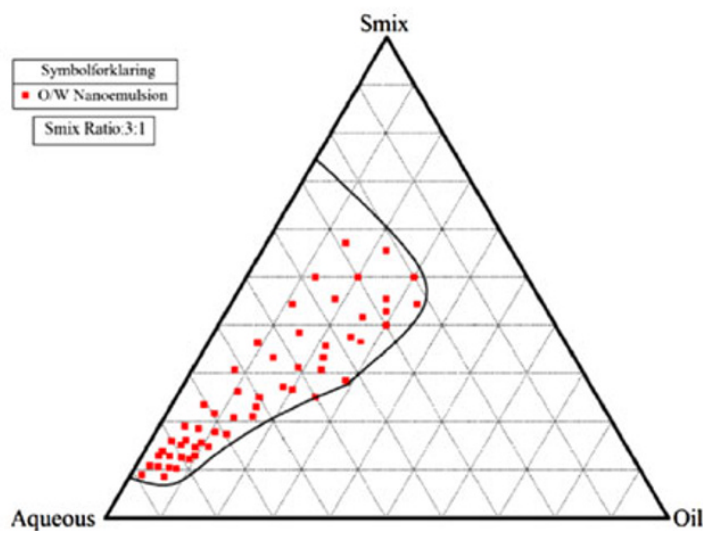

Fig. 1 Phase diagrams constructed for preparation of nanoemulsions 
Preparation of etoricoxib gel

EXB gel was prepared by dispersing the gelling agent Carbopol-934 (0.5-1.5\%) in the required quantity of distilled water and kept in the dark for $24 \mathrm{~h}$ for complete swelling. Then, $2 \% \mathrm{w} / \mathrm{w}$ of EXB in a specific quantity of the nanoemulsion solution was added slowly into this aqueous dispersion. Finally, triethanolamine (TEA) was added to obtain a homogeneous dispersion of the gel.

Thermodynamic stability studies

To overcome the problem of metastable formulations, thermodynamic stability tests were performed. The selected formulations were centrifuged at 3,500 rpm for $15 \mathrm{~min}$. The formulations that did not show any phase separation were subjected to the heating and cooling cycle. Six cycles between refrigeration temperature $\left(4^{\circ} \mathrm{C}\right)$ and $45^{\circ} \mathrm{C}$ with storage at each temperature of not less than $48 \mathrm{~h}$ were done. The formulations, which were stable at these temperatures, were further subjected to a freeze-thaw cycle between -4 and $25^{\circ} \mathrm{C}$. The formulations that survived these thermodynamic stability tests were considered for further studies (Shinoda and Kunieda 1983; DelgadoCharro et al. 1997).

\section{Droplet size analysis}

Droplet size distribution of the nanoemulsion was determined by photon correlation spectroscopy that analyzes fluctuations in light scattering due to Brownian motion of the particles, using a Zetasizer $1000 \mathrm{HS}$ (Malvern Instruments, UK). The formulation $(0.1 \mathrm{ml})$ was dispersed in $50 \mathrm{ml}$ of water, mixed thoroughly and light scattering was carried out at an angle of $90^{\circ}$ at $25^{\circ} \mathrm{C}$.

\section{Rheological studies and viscosity measurements}

The viscosity of the formulations was determined using a Brookfield Cup and Bob Viscometer (Brookfield Engineering Laboratories, Middleboro, MA) at $25 \pm 0.3{ }^{\circ} \mathrm{C}$ and $100 \mathrm{rpm}$ (Lawrence and Rees 2000).

\section{Permeation studies}

In vitro skin permeation studies were performed using porcine abdominal skin with a Franz diffusion cell having an effective diffusion area of $0.785 \mathrm{~cm}^{2}$ and $4 \mathrm{ml}$ receiver chamber capacity. Full-thickness porcine skin was excised from the abdominal region and hair was removed with an electric clipper. The subcutaneous tissue was removed surgically, and the dermis side was wiped with isopropyl alcohol to remove adhering fat. The leaned skin was washed with distilled water and stored in the deep freezer at $0{ }^{\circ} \mathrm{C}$ until further use. The skin was brought to room temperature and mounted between the donor and receiver compartments of the Franz diffusion cell, with the stratum corneum side facing the donor compartment and the dermal side facing the receiver compartment. The thickness of the skin was $50-70 \mu \mathrm{m}$ as measured with a validated micrometer screw gauge.

The receiver chamber was filled with phosphate-buffered saline (PBS) solution $\mathrm{pH} \mathrm{7.4,} \mathrm{stirred} \mathrm{with} \mathrm{a} \mathrm{magnetic}$ rotor at a speed of $50 \mathrm{rpm}$, and maintained at a temperature of $37 \pm 1{ }^{\circ} \mathrm{C}$. A quantity of the nanoemulsion diluted with $50 \%$ water was used for the release studies. Since the nanoemulsion had $2 \%(20 \mathrm{mg} / \mathrm{ml})$ of the drug, the final drug concentration in the gel was $10 \mathrm{mg} / \mathrm{ml}$ which was equivalent to the amount in the marketed formulation.

One $\mathrm{ml}$ of the optimized nanoemulsion formulation (equivalent to $10 \mathrm{mg} / \mathrm{ml}$ ETX) or $1 \mathrm{~g}$ of a conventional gel (10 mg/g) was placed in the donor compartment and sealed with paraffin film to provide occlusive conditions. Samples were withdrawn at regular intervals $(0.5,1,2,3,4,5$ and $6 \mathrm{~h}$ ) and sink conditions were maintained by replacement with fresh medium. Samples were filtered through a 0.45 $\mu \mathrm{m}$ membrane filter and analyzed for drug content by a validated HPLC method using a UV detector at $284 \mathrm{~nm}$. The HPLC system used was Jasco PU 1580 with Chrompass software. HPLC conditions included: column HiQ Sil $\mathrm{C} 8$, flow rate $1 \mathrm{ml} / \mathrm{min}$, mobile phase methanol:acetonitrile:water (55:23:32), retention time $4.2 \mathrm{~min}$. Each experiment was conducted in triplicate (Dreher et al. 1997; Gasco et al. 1991; Krielgaard 2001; Krielgaard et al. 2001; Ktistis and Niopas 1998).

\section{Histopathological examination of skin specimens}

The skin specimens subsequent to in vitro permeation studies were histopathologically examined. They were stored in $10 \%$ formalin solution. Vertical sections were dehydrated using ethanol, embedded in paraffin for fixing and stained with hematoxylin and eosin. These samples were then observed under a light microscope (Motic, Tokyo, Japan) and compared with the control sample. Three different sites of each were scanned and evaluated for possible abnormalities due to the formulation.

In vivo efficacy studies

In vivo studies were carried out as per a protocol approved by the CPCSEA and Institutional Animal Ethics Committee (IAEC). The anti-inflammatory and sustaining action of the optimized formulation was evaluated in Wistar rats by the carrageenan-induced hind paw edema method developed by Winter (1965). Formulation F9 was selected for 
the study of in vivo anti-inflammatory effects based on its higher drug permeation, lowest droplet size and lowest viscosity for better dispersion properties. Thirty rats weighing between 120 and $150 \mathrm{~g}$ were randomly categorized into five groups each containing six rats: normal, toxicant, Test 1 [nanoemulsion (F9)], Test 2 [nanoemulsion gel (NG)], and conventional gel (CG). The animals were kept under standard laboratory conditions, at $25 \pm 1{ }^{\circ} \mathrm{C}$ and relative humidity $55 \pm 5 \%$. The animals were housed in polypropylene cages, six per cage, with free access to a standard laboratory diet and water ad libitum. The dose for the rats was calculated based on the weight of the rats according to the surface area ratio (Gosh 2005). Paw edema was induced by injecting $0.1 \mathrm{ml}$ of a $1 \% \mathrm{w} / \mathrm{w}$ homogeneous suspension of carrageenan in distilled water. Control, F9, NG, and CG were applied on the hind paw region of all animals (except in normal group) half an hour before sub-planter injection of carrageenan in the right paws. The volume of paw was measured at $1,2,3,4,5,6$, and $24 \mathrm{~h}$ after injection using a digital plethysmometer and expressed as percent edema relative to initial hind paw volume. Percent inhibition of edema produced by each formulation-treated group was calculated against the respective control group. Results of anti-inflammatory activity were compared using the Dunnett's test of one-way ANOVA.

\section{Results and discussion}

The excipients interaction studies were carried out by infrared spectroscopy (Table 3) and DSC (Figs. 2, 3). The excipients selected for the formulation are pharmaceutically acceptable, nonirritating, and non-sensitizing to the skin and belong to the generally regarded as safe (GRAS) category. Higher solubility of the drug in the oil phase was another important criterion, as it would help the nanoemulsion maintain the drug in a solubilized form.

As is evident from Figs. 2 and 3, there is no interaction between the drug and selected excipients.

The surfactants were selected based on formation of the most stable mixture. This was evaluated on the basis of appearance, viscosity and droplet size. Only combinations $1: 0,1: 1,2: 1,3: 1$ (Fig. 1) produced formulations that formed effective nanoemulsions and were stable; the other ratios gave an unstable formulation and/or a narrow range of nanoemulsion formation.

\section{Nanoemulsion droplet size analysis}

The droplet size increased with the increase in concentration of oil in the formulations. All the formulations had droplets in the nano-range. Polydispersity is the ratio of standard deviation to mean droplet size and indicates the
Table 3 Comparison of IR spectra of pure drug and formulation

Fig. 2 DSC thermogram of etoricoxib

\begin{tabular}{lllll}
\hline Sr. no. & Bond & Frequency range, $\mathrm{cm}^{-1}$ & $\begin{array}{l}\text { Pure drug, } \\
\text { IR bonds cm }\end{array}$ & $\begin{array}{l}\text { Formulation, } \\
\text { IR bonds cm }\end{array}$ \\
\hline 1 & C-H & $2,962-2,853$ stretch & $2,852.82$ & $2,853.16$ \\
2 & C-C & $1,600-1,450$ stretch & $1,598.99$ & $1,593.74$ \\
3 & C-H & $1,470-1,430$ bend & $1,427.32$ & $1,430.29$ \\
4 & C-N & $1,360-1,310$ vibration & $1,309.67$ & $1,311.78$ \\
5 & S=O & $1,160-1,140$ & $1,153.43$ & $1,154.21$ \\
6 & $\mathrm{C}-\mathrm{Cl}$ & $800-600$ & 721.7 & 722 \\
\hline
\end{tabular}

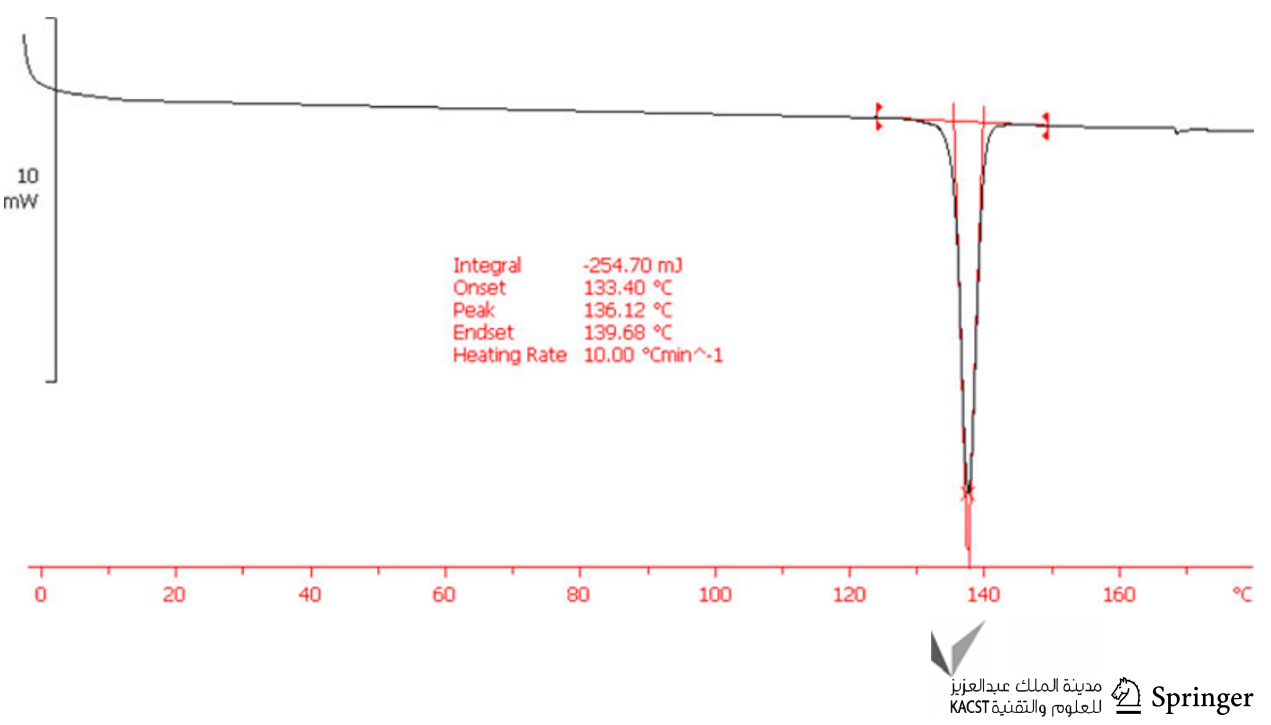


Fig. 3 DSC thermogram of formulation

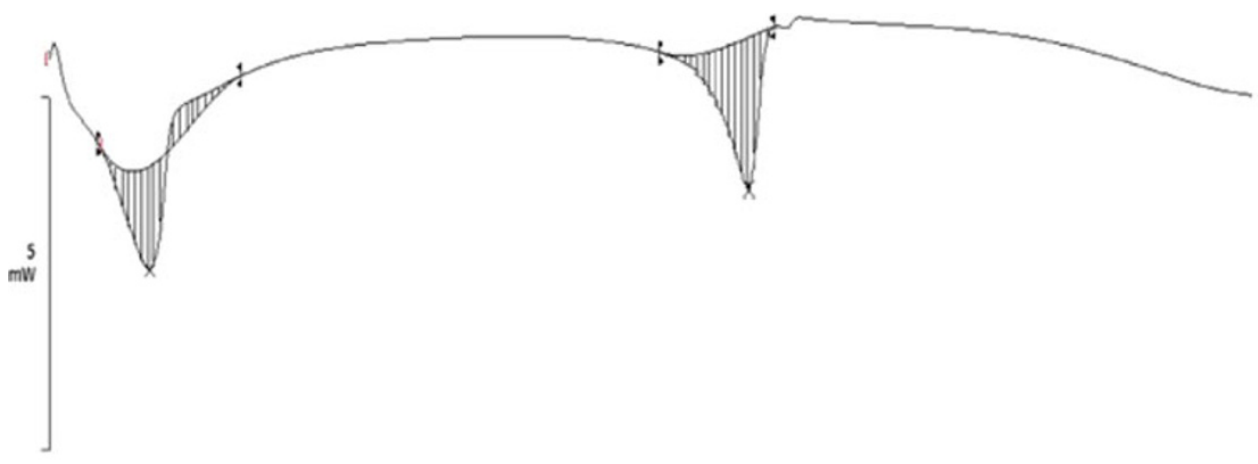

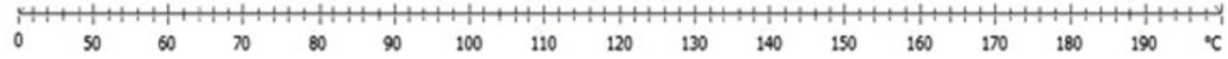

uniformity of droplet size within the formulation: higher the polydispersity, lower the uniformity of the droplet size in the formulation. Although the polydispersity values of all the formulations was very low, indicating uniformity of droplet size within each formulation, the polydispersity of formulation F9 was lowest (0.452). Also, the droplet size of formulation F9, containing $10 \%$ oil, was lowest (50 nm) (Table 4). The droplet size distribution of the optimized nanoemulsion formulation (F9) is shown in Fig. 4.

Rheological studies

Most nanoemulsions possess a very low viscosity and, therefore, their application may be inconvenient. In order

Table 4 Particle size and viscosity data of formulations

\begin{tabular}{lllr}
\hline $\begin{array}{l}\text { Formulation } \\
\text { code }\end{array}$ & $\begin{array}{l}\text { Droplet size mean } \\
(\mathrm{nm})\end{array}$ & Polydispersity & Viscosity $(\mathrm{cps})$ \\
\hline F1 & $89.33 \pm 4.04$ & $0.67 \pm 0.17$ & $90.44 \pm 5.44$ \\
F4 & $83.33 \pm 4.73$ & $0.81 \pm 0.16$ & $81.10 \pm 3.23$ \\
F9 & $50.67 \pm 6.03$ & $0.44 \pm 0.08$ & $80.06 \pm 4.19$ \\
NG & $86.67 \pm 5.51$ & $2.24 \pm 0.11$ & $3,256.69 \pm 43.41$
\end{tabular}

to overcome this disadvantage, a gelling agent such as Carbopol-934 was used to increase the viscosity of the nanoemulsion forming a nanoemulsion-based gel which is more suitable for topical application. A suitable viscosity of Carbopol-934 also results in enhanced skin permeation of the drug due to constant contact of the preparation with skin. The observed viscosities of all the formulations prepared were in range 3,000-3,500 cps making the formulated product suitable for topical administration. For some fluids, viscosity is a constant over a wide range of shear rates (Newtonian fluids). The fluids without a constant viscosity (non-Newtonian fluids) cannot be described by a single number. Non-Newtonian fluids exhibit a variety of different correlations between shear stress and shear rate. The system prepared was non-Newtonian shear thinning since the viscosity decreases with rate of shear stress.

Permeation studies

The ex vivo permeation profiles of ETX through excised steady increase in the concentration of ETX in the receptor chamber with time is observed. Statistical evaluation of the dorsal porcine skin are shown in the graph (Fig. 5). A
Fig. 4 Droplet size distribution of optimized nanoemulsion formulation (F9)

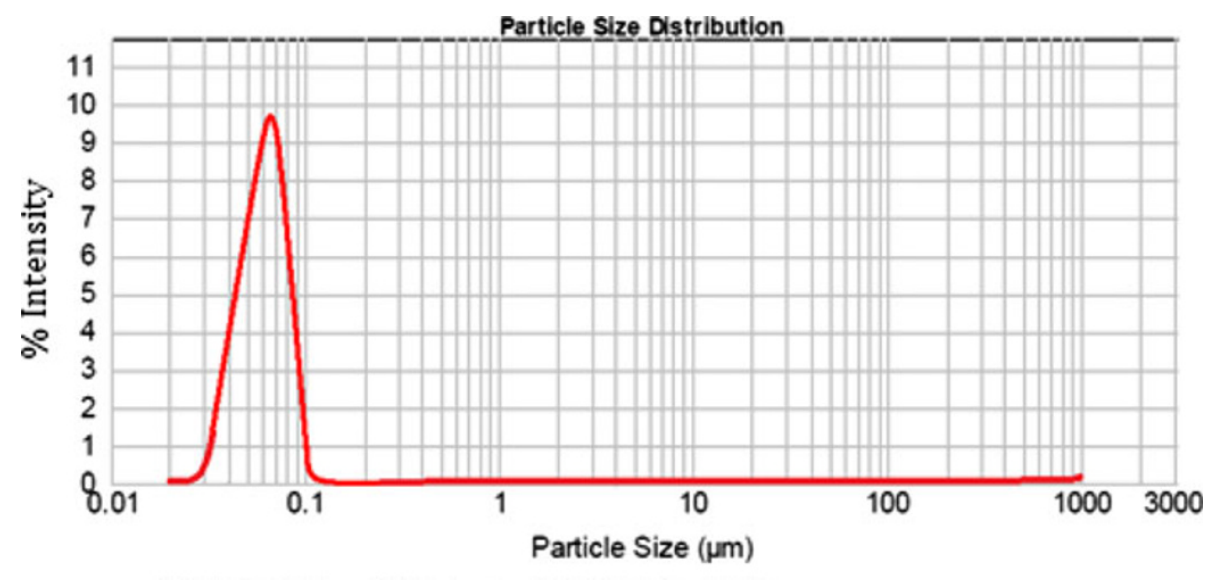

— Nilesh EXB Form 7, Wendesday 17, 2011 5:38:45 PM 


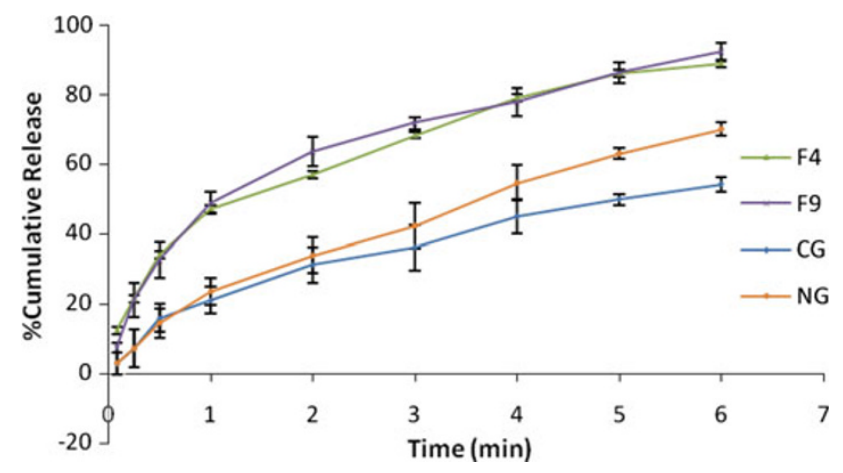

Fig. 5 Ex vivo permeation studies on nanoemulsion (NE) $(F 4, F 9)$, nanoemulsion gel $(N G)$ and conventional gel $(C G)$

flux throughout the $6 \mathrm{~h}$ of study showed that among all the formulations, nanoemulsions F4 and F9 exhibited maximum permeation (above $90 \%$ ) at the end of $6 \mathrm{~h}$, while the cumulative permeation of ETX from the nanoemulsionbased gel $(\mathrm{NG})$ and conventional gel $(\mathrm{CG})$ containing the same amount of ETX were 80 and $70 \%$, respectively, $6 \mathrm{~h}$ post-application. Higher release was obtained with the fluid nanoemulsions than the viscous gels due to increased penetration through the membrane.

Histopathological studies

The photomicrographs of untreated porcine skin (control) showed normal skin with well-defined epidermal and dermal layers (Fig. 6).

Skin appendages were within normal limits (Fig. 6A, $\mathrm{B})$, and skin was treated with nanoemulsion formulation (F9, NG) for $24 \mathrm{~h}$ (Fig. 6C, D).

These observations support the compatibility of the formulation with skin. There were no apparent signs of skin irritation (erythema and edema) observed on visual examination of the skin specimens treated with nanoemulsion formulations indicating absence of any skin irritation as a consequence of nanoemulsion treatment.

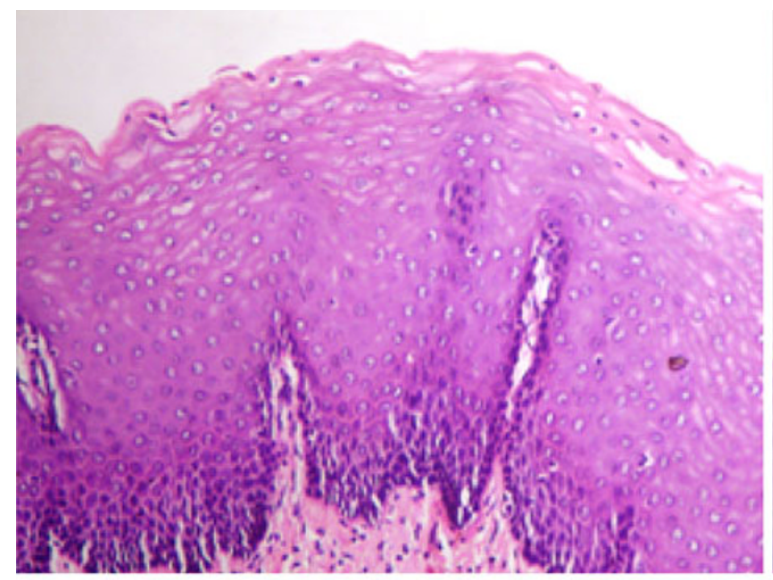

A Untreated 1

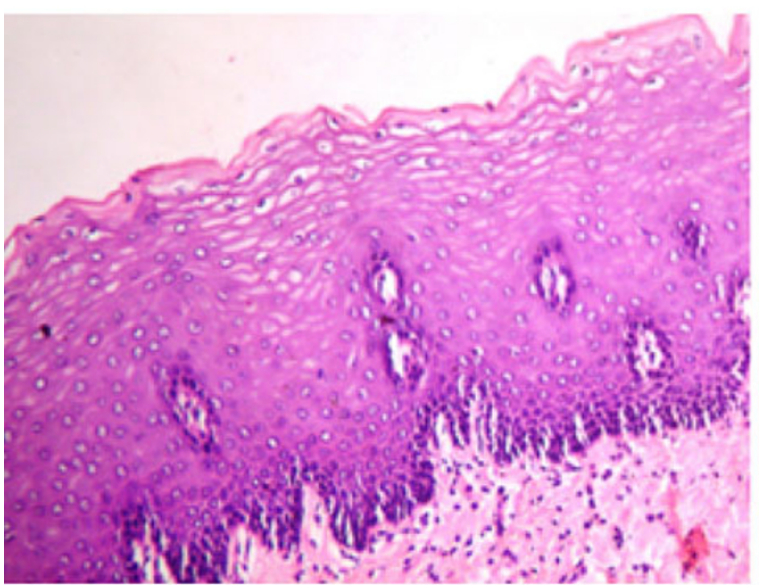

C Treated with Nano F9

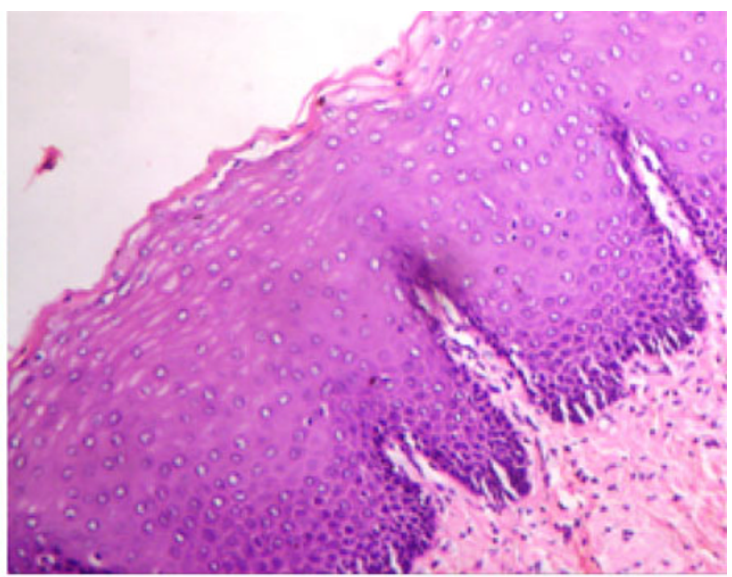

B untreated 2

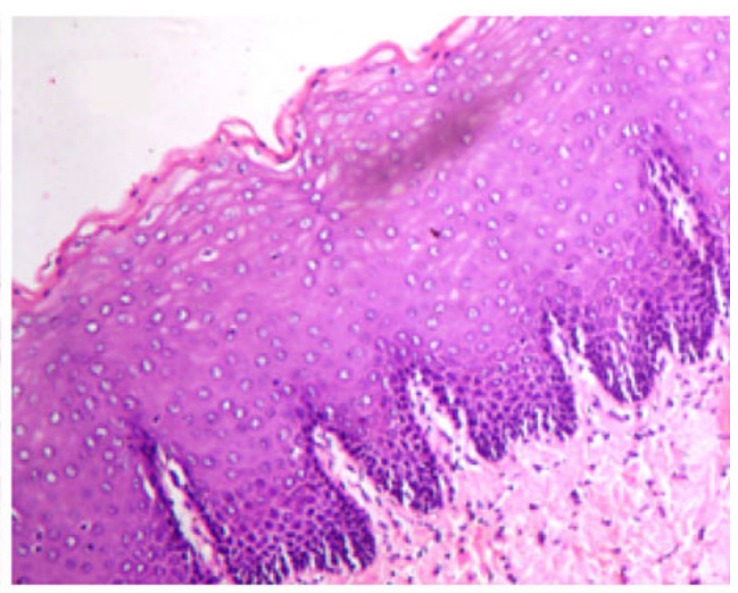

D Treated with Nano F9

Fig. 6 Photomicrograph of histopathological analysis of porcine abdominal skin 


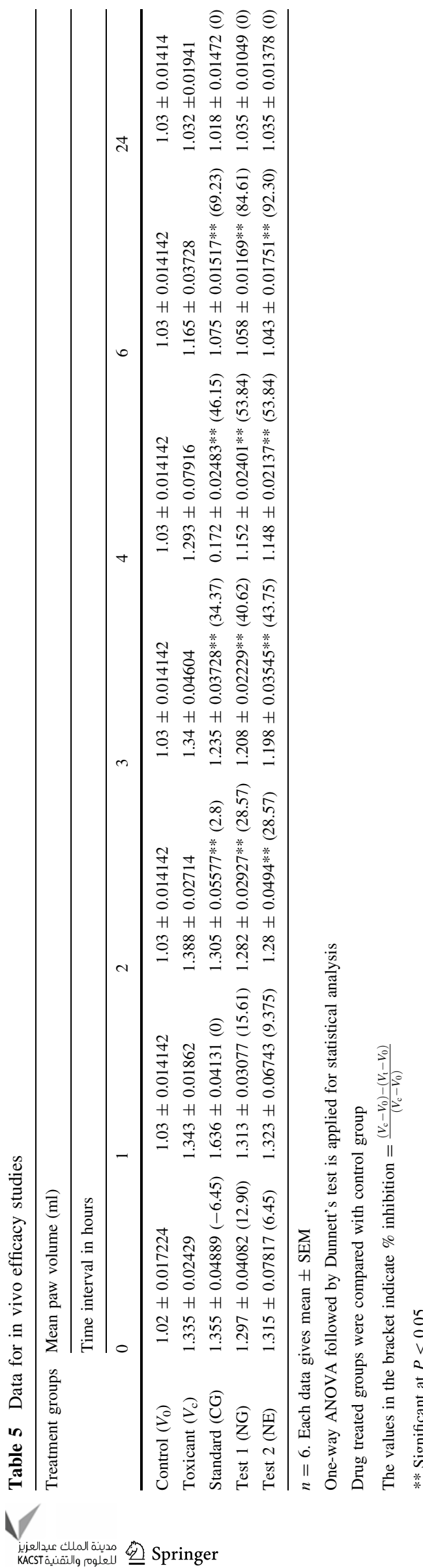

In vivo efficacy studies

The percent inhibition values $6 \mathrm{~h}$ post-administration were found to be high for nanoemulsion formulations (F4, F9) as compared with conventional gel (CG); this difference was significant $(P<0.05)$. The percent inhibition values for the formulations are shown in Table 5.

\section{Skin irritation test}

No erythema was observed on visual inspection after application of ETX nanoemulsion formulations on rat skin. Thus, the developed formulation is non-sensitizing and safe for use.

\section{Conclusion}

In this work, a nanoemulsion-based gel formulation with suitable viscosity for topical administration has been developed to deliver anti-inflammatory agents such as etoricoxib. The optimized formulation contained $20 \%$ of oil phase (Triacetin), $38 \%$ of surfactant mixture (Cremophor RH 40 and Transcutol P) and $42 \%$ of distilled water. From the studies, it is observed that the formulated nanoemulsions and nanoemulsion gel released up to 90 and $80 \%$ of the drug, respectively, as compared to $70 \%$ from the conventional gel product. The formulation was nonsensitizing and safe for use prepared with nonirritating, pharmaceutically acceptable ingredients. No additional permeation enhancers were needed to be added since the excipients themselves acted as permeation enhancers. A high percent inhibition of edema was observed with the nanoemulsion $(92.30 \%)$ and nanoemulsion gel $(84.61 \%)$ as compared with the conventional gel $(69.23 \%)$. Thus, it can be concluded that the developed nanoemulsion-based gels have a greater potential for topical drug delivery as compared to conventional formulations.

Open Access This article is distributed under the terms of the Creative Commons Attribution License which permits any use, distribution, and reproduction in any medium, provided the original author(s) and the source are credited.

\section{References}

Alvarez-Figueroa MJ, Blanco-Mendez J (2001) Transdermal delivery of methotrexate: iontophoretic delivery from hydrogels and passive delivery from microemulsions. Int J Pharm 215:57-65

Barzin J, Sadatnia B (2007) Theoretical phase diagram calculation and membrane morphology, evaluation for water/solvent/polyethersulfone systems. Polymer 48:1620-1631

Craig DQM, Barker SA, Banning D, Booth SW (1995) An investigation into the mechanisms of self-emulsification using 
particle size analysis and low frequency dielectric spectroscopy. Int J Pharm 114:103-110

Delgado-Charro MB, Iglesias-Vilas G, Blanco-Mendez J, LopezQuintela MJ, Marty MA, Guy JP (1997) Delivery of a hydrophilic solute through the skin from novel microemulsion systems. Eur J Pharm Biopharm 43:37-42

Dreher F, Walde P, Walter P, Wehrli E (1997) Interaction of a lecithin microemulsion gel with human stratum corneum and its effect on transdermal transport. J Control Release 45:131-140

Escribano E, Calpena AC, Queralt J, Obach R, Domenech J (2003) Assessment of diclofenac permeation with different formulations: anti-inflammatory study of a selected formula. Eur J Pharm Sci 19:203-210

Gasco MR, Gallarate M, Pattarino F (1991) In vitro permeation of azelaic acid from viscosized microemulsions. Int $\mathrm{J}$ Pharm 69:193-196

Gaurel A, Martel AM, Castaner J (1997) Celecoxib, anti-inflammatory, cyclo-oxygenase-2 inhibitor. Drug Future 22:711-714

Gonzalez E, Cruz C, Nicolas R, Egido J, Herrero-Beaumont G (1994) Long-term effects of nonsteroidal anti-inflammatory drugs on the production of cytokines and other inflammatory mediators by blood cells of patients with osteoarthritis. Agents Actions 41: $171-178$

Gosh MN (2005) Fundamentals of experimental pharmacology, vol 3. Hilton and Co., Kolkata, p 192

Kemken J, Ziegler A, Muller BW (1992) Influence of supersaturation on the pharmacodynamic effect of bupranolol after dermal administration using microemulsions as vehicle. Pharm Res 9:554-558

Kreilgaard M (2001) Dermal pharmacokinetics of microemulsion formulations determined by in vitro microdialysis. Pharm Res 18:367-373

Kreilgaard M, Pedersen EJ, Jaroszewski JW (2000) NMR characterization and transdermal drug delivery potentials of microemulsion systems. J Control Release 69:421-433

Kreilgaard M, Kemme MJB, Burggraaf J, Schoemaker RC, Cohen AF (2001) Influence of a microemulsion vehicle on cutaneous bioequivalence of a lipophilic model drug assessed by microdialysis and pharmacodynamics. Pharm Res 18:593-599

Kriwet K, Muller-Goymann CC (1995) Diclofenac release from phospholipids drug systems and permeation through excised human stratum corneum. Int J Pharm 125:231-242

Ktistis G, Niopas I (1998) A study on the in vitro percutaneous absorption of propranolol from disperse systems. J Pharm Pharmacol 50:413-419
Lawrence MJ, Rees GD (2000) Microemulsion-based media as novel drug delivery systems. Adv Drug Deliv Rev 45:89-121

Lee PJ, Langer R, Shastri VP (2003) Novel microemulsion enhancer formulation for simultaneous transdermal delivery of hydrophilic and hydrophobic drugs. Pharm Res 20:264-269

Lehmann L, Keipert S, Gloor M (2001) Effects of nanoemulsions on the stratum corneum and hydrocortisone penetration. Eur $\mathrm{J}$ Pharm Biopharm 52:129-136

Li P, Ghosh A, Wagner RF, Joshi YM, Serajuddin ATM (2005) Effect of combined use of nonionic surfactant on formation of oil-inwater microemulsions. Int J Pharm 288:27-34

Osborne DW, Ward AJ, Neil KJ (1991) Microemulsions as topical delivery vehicles: in vitro transdermal studies of a model hydrophilic drug. J Pharm Pharmacol 43:450-454

Peltola S, Saarinen P, Kiesvaara J, Suhonen T, Urtti A (2003) Nanoemulsions for topical delivery of estradiol. Int $\mathrm{J}$ Pharm 254:99-107

Rhee YS, Choi JG, Park ES, Chi SC (2001) Transdermal delivery of ketoprofen using microemulsions. Int J Pharm 228:161-170

Shafiq S, Faiyaz S, Sushma T, Ahmad FJ, Khar RK, Ali M (2000) Development and Bioavailability assessment of ramipril nanoemulsion formulation. Eur J Pharm Biopharm 66:227-243

Shafiq S, Faiyaz S, Sushma T, Ahmad FJ, Khar RK, Ali M (2007) Design and development of oral oil in water ramipril nanoemulsion formulation: in vitro and in vivo evaluation. $\mathrm{J}$ Biomed Nanotech 3:28-44

Shinoda K, Kunieda H (1983) Phase properties of emulsions: PIT and HBL. In: Schuster D (ed) Encyclopedia of emulsion technology. Marcel Dekker, New York, pp 337-367

Trotta M (1999) Influence of phase transformation on indomethacin release from microemulsions. J Control Release 60:399-405

Walters KA (1989) Penetration enhancers and their use in transdermal therapeutic systems. In: Hadgraft J, Guy RH (eds) Transdermal drug delivery, developmental issues and research initiatives. Marcel Dekker, New York, pp 197-246

Winter CA (1965) Antiinflammatory testing methods: comparative evaluation of indomethacin and other agents. NSAID 82: 190-202

Yamazaki R, Kawai S, Mastsuzaki T et al (1997) Aceclofenac blocks prostaglandin E2 production following its intracellular conversion into cyclooxygenase inhibitors. Eur J Pharmacol 329:181-187

Yang JH, Kim Y, Kim KM (2002) Preparation and evaluation of aceclofenac microemulsion for transdermal delivery system. Arch Pharm Res 25:534-540 